\title{
Quality, Yield and Economics of Rabi Groundnut as Influenced By Irrigation Scheduling and Phosphogypsum Levels
}

\author{
R. Naresha* ${ }^{*}$ P. Laxminarayana, K.B. Suneetha Devi and V. Sailaja
}

Professor Jayashankar Telangana State Agricultural University, Department of Agronomy, College of Agriculture, Rajendranagar, Hyderabad-500030, Telangana State, India

*Corresponding author

\section{A B S T R A C T}

\begin{tabular}{|l|}
\hline K e y w o r d s \\
Phosphogypsum, \\
Irrigation scheduling, \\
Economics of rabi \\
Groundnut, Quality of \\
groundnut
\end{tabular}

\section{Introduction}

Groundnut is an important oil and protein source to a large portion of the population in India. It is an annual, herbaceous legume and considered as king of vegetable oil seed crops in India and occupies a pre-eminent position in national edible oil economy. Groundnut seed contain 47-53 per cent oil, 26 per cent protein and 11.5 per cent starch. It is currently grown in an area of 42 Million hectares over the globe. Cultivation of groundnut under rainfed conditions and imbalanced nutrient management are the main reasons for low productivity of groundnut in Andhra Pradesh.
Irrigation water, a crucial input in crop production is scarce and expensive. Efficient use of this input is essential which can be achieved through judicious water management practices. Adequate and timely supply of water is essential for higher yields. Keeping the total quantity of irrigation water constant, increasing the frequency of irrigation would maximize the yield in groundnut (Giri et al., 2017).

Groundnut is grown during rainy, winter and summer seasons in India. The average productivity is relatively low in rainy season. Groundnut has specific moisture needs due to 
its peculiar feature of producing pods underground. In groundnut early moisture stress restricts the vegetative growth which in turn reduces the yield and at the peak flowering and pegging period is most sensitive as the peg cannot penetrate through dry and hard surface. The rabi crop avails the residual moisture and the scanty rainfall during winter and produces substantial yield as compared to the kharif crop and few supplementary irrigations would improve the yield. Because of high productivity under assured irrigation, groundnut cultivation in rabi season is gaining popularity in irrigation scheduling, a climatologically approach based on IW/CPE ratio (IW- irrigation water, CPE- Cumulative pan evaporation) has been found most appropriate. This approach integrates all the weather parameters that determine water use by the crop and is likely to increase production at least $15-20 \%$. Optimum scheduling of irrigation led to increase in pod yield and water use efficiency (WUE) (Taha and Gulati, 2001).

To ensure increased yields of rabi groundnut in traditional areas of Andhra Pradesh and Telangana it is necessary to have a thorough understanding of the changes in the soil-plantwater relations and various morphophysiological processes in relation to scheduling of irrigation water. Studies on various aspects of groundnut nutrition are limited particularly under varied soil moisture regimes, hence efforts are needed to quantify the crop response vis-a-visat different nutrient levels.

Among the sources of sulphur, phosphogypsum is cheaper and potential source. It is a solid waste by-product of the wet phosphoric acid production from rock phosphate. Phosphogypsum contains 16 per cent sulphur and 21 per cent calcium along with meagre amount of phosphorus (0.2-1.2 \% $\left.\mathrm{P}_{2} \mathrm{O}_{5}\right)$ and trace amounts of silica $\left(\mathrm{SiO}_{2}\right)$, iron
$\left(\mathrm{Fe}_{2} \mathrm{O}_{3}\right)$, aluminium $\left(\mathrm{Al}_{2} \mathrm{O}_{3}\right)$, sodium $\left(\mathrm{Na}_{2} \mathrm{O}\right)$, potassium $\left(\mathrm{K}_{2} \mathrm{O}\right)$ and some heavy metals (Biswas and Sharma, 2008). Approximately 5.5 Million tonnes of phosphogypsum is discharged for every one Million tonnes of phosphoric acid production. In India, 6 to 8 Million tonnes of phosphogypsum is produced annually which supplies 1 to 1.5 Million tonnes of sulphur and 1.5 to 2 Million tonnes of calcium.

The phosphogypsum, unlike other sulphur sources, offers all desirable agronomic features of an efficient sulphur fertilizer besides supplying calcium that is readily available to the growing plant, while elemental sulphur and organic sulphur must undergo microbial conversion before sulphur is made available to plants, but the sulphur in phosphogypsum becomes readily available in sulphate form. At the same time, sulphate form is kept available for a longer period due to its low solubility in water. Most of the other sulphate salts that are used for fertilizer are highly soluble and the sulphate may be leached from the soil before the plant removal (Biswas and Sharma, 2008).

In spite of additional nutritional value and desirable agronomic features, a high proportion is either dumped or staked for increasing concern to the risk of exposure to radiation.

However, the relative radiation risk to people or the environment falls significantly below the level of radiation to which we are exposed through Naturally Occurring Radioactive Material (NORM).

Hence, it may not be prudent, therefore, to allow such wastage of this large sulphur and calcium rich by-product $(16 \% \mathrm{~S}$ and $21 \% \mathrm{Ca})$ in the back drop of wide spread sulphur and calcium deficiencies in Indian soils (Biswas and Sharma, 2008). Keeping this in view, this 
study was undertaken to investigate the influence of moisture regimes and phosphogypsum levels on growth and yield of rabi groundnut.

\section{Material and Methods}

Field experiment was conducted at College farm, Acharya N. G. Ranga Agricultural University, Rajendranagar, Hyderabad during rabi 2013-14 on sandy loam soil having low organic carbon $(0.53 \%)$ and available nitrogen (238.33 kg ha $\left.{ }^{-1}\right)$, medium available phosphorous (29.33 kg ha $\mathrm{kg}^{-1}$, sulphur and calcium (14.30 and $10.00 \mathrm{~kg} \mathrm{ha}^{-1}$ ), high potassium (423.36 kg ha ${ }^{-1}$ ) and neutral in reaction.

The experiment was laid out in split plot design with combinations of three moisture regimes viz., 0.6, 0.8 and $1.0 \mathrm{IW} / \mathrm{CPE}$ ratios and five phosphogypsum fertilizer levels viz., $\mathrm{Pg}_{1}$ : Control $\left(500 \mathrm{~kg} \mathrm{ha}^{-1}\right.$ gypsum at flower initiation), $\mathrm{Pg}_{2}$ : Phosphogypsum @ $250 \mathrm{~kg}$ $\mathrm{ha}^{-1} \quad$ (at flower initiation), $\mathrm{Pg}_{3}$ : Phosphogypsum @ $250 \mathrm{~kg} \mathrm{ha}^{-1}(1 / 2$ as basal and $1 / 2$ at flower initiation), $\quad \mathrm{Pg}_{4}$ : Phosphogypsum @ $\quad 500 \quad \mathrm{~kg}$ $\mathrm{ha}^{-1}(1 / 2$ as basal and $1 / 2$ at flower initiation), $\mathrm{Pg}_{5}$ : Phosphogypsum @ $500 \mathrm{~kg} \mathrm{ha}^{-1}$ (at flower initiation) and was replicated thrice. Groundnut variety K-6 (Kadiri-6) was sown on 10-10-2013 at a spacing of $22.5 \mathrm{~cm} \mathrm{x} 10$ $\mathrm{cm}$ with one seed hill ${ }^{-1}$. Recommended N P K applied to all the treatments uniformly @ 30: 50: $50 \mathrm{~kg} \mathrm{ha}^{-1}$. Nitrogen and Phosphorus applied through urea and DAP, potassium through muriate of potash. Whole quantity of phosphorus and potassium and $1 / 2$ nitrogen applied as basal and remaining $1 / 2$ Nitrogen as top dressing at 25-30 DAS. Mean maximum and minimum temperatures were $32.8^{\circ} \mathrm{C}$ and $22.1^{\circ} \mathrm{C}$ respectively and $282.2 \mathrm{~mm}$ rainfall was received in 11 rainy days during the crop growing period. Mean bulk density and total available soil moisture in $60 \mathrm{~cm}$ depth of soil was $1.6 \mathrm{~g} \mathrm{~cm}^{-3}$ and $127.6 \mathrm{~mm}$ respectively. Mean moisture percentage at field capacity and permanent wilting point was 19.2 and 5.9. The total applied irrigation water was 267, 222 and 178 ha.mm for IW/CPE ratio of 1.0, 0.8 and 0.6 respectively. Five, four and three irrigations were given to IW/CPE ratio of 1.0, 0.8 and 0.6 respectively along with one irrigation to all treatments one day before harvesting. For every irrigation, $50 \mathrm{~mm}$ of water was applied using water meter in closed channels. Daily readings of evaporation were recorded from USWB class "A" open pan evaporimeter and irrigations were scheduled based on IW/CPE ratios.

Yield attributes and yield were recorded at harvest, while quality and economics were recorded after harvest of groundnut. Statistical analysis was done to all the recorded data as per Panse and Sukhatme (1985).

\section{Results and Discussion}

The data pertaining to yield attributes as influenced by moisture regimes and phosphogypsum levels was presented in Table 1. Yield attributes viz., number of pods plant ${ }^{-1}$, 100 kernel weight (g), shelling percentage were significantly influenced by moisture regimes and phosphogypsum levels were higher at $\mathrm{I}_{3}(1.0 \mathrm{IW} / \mathrm{CPE})$ and was on par at $\mathrm{I}_{2}$ (0.8 IW/CPE) for number of pods plant ${ }^{-1}, 100$ kernel weight (g), shelling percentage. Frequent irrigation under $I_{3}$ treatment might have created favorable moisture conditions for the crop growth consequently increased the values of the yield attributes than other treatments $\left(I_{1}\right.$ and $\left.I_{2}\right)$. These results are in close conformity with the findings of Santosh Behera et al., (2015), Patel et al., (2009), Dey et al., (2007) and Shaikh et al., (2004).

Among the phosphogypsum levels, application of phosphogypsum @ $500 \mathrm{~kg} \mathrm{ha}^{-1}$ at flower initiation recorded significantly 
higher yield attributes and was statistically on par with application of gypsum @ $500 \mathrm{~kg} \mathrm{ha}^{-1}$ at flower initiation for all yield attributes except number of pods plant ${ }^{-1}$. Interaction between moisture regimes and phosphogypsum levels was significant only with number of pods plant ${ }^{-1}$. Significantly highest number of pods plant ${ }^{-1}$ (15.8) noticed at interaction of $\mathrm{I}_{3}(1.0 \quad \mathrm{IW} / \mathrm{CPE})$ and phosphogypsum @ $500 \mathrm{~kg} \mathrm{ha}^{-1}$ at flower initiation $\left(\mathrm{Pg}_{5}\right)$ followed by $\mathrm{I}_{2} \mathrm{Pg}_{5}$ and $\mathrm{I}_{3} \mathrm{Pg}_{2}$ respectively. Lowest number of pods plant $^{-1}$ (12.2) recorded at interaction of $\mathrm{I}_{1} \mathrm{Pg}_{3}(0.6$ IW/CPE) (phosphogypsum @ $250 \mathrm{~kg} \mathrm{ha}^{-1} 1 / 2$ as basal and $1 / 2$ at flower initiation) (Table 1.b). The marked improvement in yield attributes might be due to balanced nutrition and efficient and greater partitioning of metabolites and adequate translocation of nutrients to the developing reproductive parts resulting in the production of greater pod number and shelling percentage These results are in close conformity with the findings of Surendra singh and Singh (2016), Somnath chattopaddhyay and goutam kumar ghosh (2012), Rout and Jena (2009) and Dey et al., (2007).

The data pertaining to groundnut pod and haulm yield were presented in Table 1 and fig.1. The highest pod and haulm yields (21.5 and $38.4 \mathrm{q} \mathrm{ha}^{-1}$ ) were recorded when irrigation was scheduled at $\mathrm{I}_{3}(1.0 \mathrm{IW} / \mathrm{CPE})$, which was on par with $\mathrm{I}_{2}(0.8 \mathrm{IW} / \mathrm{CPE})$ treatment and both were significantly superior over $\mathrm{I}_{1}$ level of moisture regimes where in lowest yields (17.4 and $32.3 \mathrm{q} \mathrm{ha}^{-1}$ ) were recorded. The higher pod and haulm yields with more frequent irrigation $\left(\mathrm{I}_{3}\right)$ might have accounted for their favorable influence on the yield attributing characters viz. number of pods per plant, number of kernels pod ${ }^{-1}$ and 100 kernel weight.

Pod and haulm yield of groundnut was significantly increased with increase in the frequency of irrigation which was ascribed to adequate moisture availability in turn have favoured congenial conditions for the luxurious growth of crop and consequently increased the values of the yield attributes with $I_{3}$ compare to $I_{2}$ and $I_{1}$ treatments. These results are in close conformity with the findings of Santosh Behera et al., (2015), Suresh et al., (2013) and Shaikh et al., (2004).

Effect of phosphogypsum levels on pod and haulm yields were presented in Table 1 and fig. 2. Among the phosphogypsum levels, application of phosphogypsum @ $500 \mathrm{~kg} \mathrm{ha}^{-1}$ at flower initiation were recorded significantly highest pod and haulm yields (21.4 and $37.6 \mathrm{q}$ $\mathrm{ha}^{-1}$ ) whereas haulm yield was on par with gypsum application@ $500 \mathrm{~kg} \mathrm{ha}^{-1}$ at flower initiation. Lowest pod and haulm yields (17.9 and $34.4 \mathrm{q} \mathrm{ha}^{-1}$ ) were recorded under $\mathrm{Pg}_{3}$ (phosphogypsum @ $250 \mathrm{~kg} \mathrm{ha}^{-1} 1 / 2$ as basal and $1 / 2$ at flower initiation) treatment. Phosphogypsum application @ $500 \mathrm{~kg} \mathrm{ha}^{-1}$ at flowering stage might have ensured adequate supply of calcium and sulphur, have favoured for obtaining good yield.

The results of the experiment clearly suggest that phosphogypsum is also efficient in increasing the pod and yield of groundnut similar to that of gypsum.

The higher pod and haulm yield with application of phosphogypsum @ $500 \mathrm{~kg} \mathrm{ha}^{-1}$ might attributed for their favorable influence on the yield. As phosphogypsum has relatively low solubility as compared to highly soluble sulphur carriers, availability of sulphur is made for a longer period. These results are in close conformity with the findings of Surendra Singh and Singh (2016), Somnath Chattopaddhyay and Goutam Kumar Ghosh (2012), Rout and Jena (2009).

The interaction effect of moisture regimes and phosphogypsum levels on pod and haulm yields were presented in Table 1.a. and fig. 3. 
Table.1 Yield and yield attributing characters of rabi groundnut as influenced by moisture regimes and phosphogypsum levels

\begin{tabular}{|c|c|c|c|c|c|}
\hline \multirow[b]{2}{*}{ TREATMENTS } & \multicolumn{3}{|c|}{ YIELD ATTRIBUTES } & \multicolumn{2}{|c|}{ Yield $\left(\mathrm{kg} \mathrm{ha}^{-1}\right)$} \\
\hline & $\begin{array}{l}\text { No. of } \\
\text { Pods } \\
\text { Plant }^{-1}\end{array}$ & $\begin{array}{l}100 \text { Kernel } \\
\text { Weight }(g)\end{array}$ & $\begin{array}{l}\text { Shelling } \\
\%\end{array}$ & Pod yield & Haulm Yield \\
\hline \multicolumn{6}{|l|}{ MOISTURE REGIMES (I) } \\
\hline $\mathrm{I}_{1}-0.6 \mathrm{IW} / \mathrm{CPE}$ & 13.0 & 40.1 & 64.7 & 1742 & 3234 \\
\hline $\mathrm{I}_{2}-0.8 \mathrm{IW} / \mathrm{CPE}$ & 13.7 & 41.0 & 68.0 & 2081 & 3719 \\
\hline I-1.0 IW/CPE & 14.1 & 41.4 & 69.8 & 2147 & 3799 \\
\hline SEm \pm & 0.1 & 0.2 & 0.8 & 18 & 28 \\
\hline $\mathrm{CD}(\mathrm{P}=0.05)$ & 0.5 & 0.8 & 3.2 & 73 & 109 \\
\hline \multicolumn{6}{|l|}{ PHOSPHOGYPSUM LEVELS (Pg) } \\
\hline $\mathrm{Pg}_{1}$-Gypsum @ $500 \mathrm{~kg} \mathrm{ha}^{-1}$ at flower initiation & 13.7 & 41.3 & 68.2 & 2060 & 3665 \\
\hline $\mathrm{Pg}_{2}$-PG @ $250 \mathrm{~kg} \mathrm{ha}^{-1}$ at flower initiation & 13.3 & 40.2 & 66.8 & 1958 & 3556 \\
\hline 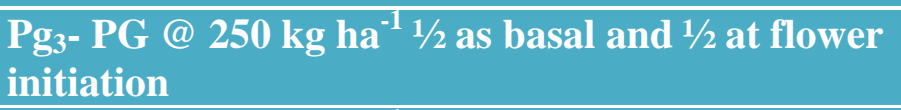 & 13.0 & 40.0 & 64.9 & 1795 & 3444 \\
\hline $\begin{array}{l}\mathrm{Pg}_{4^{-}} \text {PG @ } 500 \mathrm{~kg} \mathrm{ha}^{-1} 1 / 2 \text { as basal and } 1 / 2 \text { at flower } \\
\text { initiation }\end{array}$ & 13.4 & 40.7 & 67.1 & 2001 & 3555 \\
\hline $\mathrm{Pg}_{5}$ PG @ $500 \mathrm{~kg} \mathrm{ha}^{-1}$ at flower initiation & 14.6 & 42.1 & 70.6 & 2136 & 3701 \\
\hline SEm \pm & 0.1 & 0.4 & 0.8 & 20 & 55 \\
\hline $\mathrm{CD}(\mathrm{P}=\mathbf{0 . 0 5})$ & 0.4 & 1.0 & 2.4 & 59 & 161 \\
\hline \multicolumn{6}{|l|}{ INTERACTION (I x Pg) } \\
\hline \multicolumn{6}{|l|}{ Sub treatments at same level of main treatments } \\
\hline SEm \pm & 0.3 & 0.5 & 1.9 & 41 & 62 \\
\hline $\mathrm{CD}(\mathrm{P}=0.05)$ & 0.8 & NS & NS & 36 & 287 \\
\hline \multicolumn{6}{|l|}{ Main treatments at same level of sub treatments } \\
\hline SEm \pm & 0.3 & 0.6 & 1.5 & 111 & 90 \\
\hline $\mathrm{CD}(\mathrm{P}=0.05)$ & 0.8 & NS & NS & 116 & 270 \\
\hline
\end{tabular}


Table.1a Pod Yield and haulm yield $\left(\mathrm{kg} \mathrm{ha}^{-1}\right)$ of rabi groundnut as influenced by interaction between moisture regimes and phosphogypsum levels

\begin{tabular}{|c|c|c|c|c|c|c|c|c|}
\hline \multirow{3}{*}{ PHOSPHOGYPSUM LEVELS } & \multicolumn{4}{|c|}{ Pod yield $\left(\mathrm{kg} \mathrm{ha}^{-1}\right)$} & \multicolumn{4}{|c|}{ Haulm yield $\left(\mathrm{kg} \mathrm{ha}^{-1}\right)$} \\
\hline & \multicolumn{3}{|c|}{ MOISTURE REGIMES } & \multirow[t]{2}{*}{ MEAN } & \multicolumn{3}{|c|}{ MOISTURE REGIMES } & \multirow[b]{2}{*}{ MEAN } \\
\hline & $\begin{array}{c}\mathbf{I}_{1}-\mathbf{0 . 6} \\
\text { IW/CPE }\end{array}$ & $\begin{array}{c}\mathbf{I}_{2}-\mathbf{0 . 8} \\
\text { IW/CPE }\end{array}$ & $\begin{array}{c}\mathbf{I}_{3}-1.0 \\
\text { IW/CPE }\end{array}$ & & $\mathrm{I}_{1}-\mathbf{0 . 6} \mathrm{IW} / \mathrm{CPE}$ & $\mathrm{I}_{2}-0.8 \mathrm{IW} / \mathrm{CPE}$ & $\begin{array}{c}\mathbf{I}_{3}-1.0 \\
\text { IW/CPE }\end{array}$ & \\
\hline $\mathrm{Pg}_{1}-\mathrm{Gypsum} @ 500 \mathrm{~kg} \mathrm{ha}^{-1}$ at flower initiation & 1820 & 2134 & 2224 & 2060 & 3351 & 3822 & 3821 & 3665 \\
\hline $\mathbf{P g}_{2}-\mathrm{PG} @ 250 \mathrm{~kg} \mathrm{ha}^{-1}$ at flower initiation & 1634 & 2064 & 2175 & 1958 & 3046 & 3641 & 3982 & 3556 \\
\hline $\mathrm{Pg}_{3^{-}} \mathbf{P G} @ 250 \mathrm{~kg} \mathrm{ha}^{-1 / 1 / 2}$ as basal and $1 / 2$ at flower initiation & 1560 & 1848 & 1978 & 1795 & 3262 & 3371 & 3698 & 3444 \\
\hline $\mathrm{Pg}_{4-}$ PG @ $500 \mathrm{~kg} \mathrm{ha}^{-1 / 1 / 2}$ as basal and $1 / 2$ at flower initiation & 1788 & 2162 & 2054 & 2001 & 3100 & 3775 & 3789 & 3555 \\
\hline $\mathrm{Pg}_{5^{-}}$PG @ $500 \mathrm{~kg} \mathrm{ha}^{-1}$ at flower initiation & 1908 & 2197 & 2303 & 2136 & 3411 & 3985 & 3706 & 3701 \\
\hline MEAN & 1742 & 2081 & 2147 & 1990 & 3234 & 3719 & 3799 & 3584 \\
\hline \multicolumn{9}{|l|}{ Sub treatments at same level of main treatments } \\
\hline SEm \pm & & & & 41 & & & & 62 \\
\hline $\mathrm{CD}(\mathrm{P}=0.05)$ & & & & 111 & & & & 287 \\
\hline \multicolumn{9}{|l|}{ Main treatments at same level of sub treatments } \\
\hline SEm \pm & & & & 36 & & & & 90 \\
\hline $\mathrm{CD}(\mathrm{P}=0.05)$ & & & & 116 & & & & 270 \\
\hline
\end{tabular}

Table.1b Number of pods plant ${ }^{-1}$ and Oil content (\%) of rabi groundnut as influenced by interaction between moisture regimes and phosphogypsum levels

\begin{tabular}{|c|c|c|c|c|c|c|c|c|}
\hline \multirow{3}{*}{ PHOSPHOGYPSUM LEVELS } & \multicolumn{4}{|c|}{ Number of pods plant $^{-1}$} & \multicolumn{4}{|c|}{ Oil content } \\
\hline & \multicolumn{3}{|c|}{ MOISTURE REGIMES } & \multirow[t]{2}{*}{ MEAN } & \multicolumn{3}{|c|}{ MOISTURE REGIMES } & \multirow[t]{2}{*}{ MEAN } \\
\hline & $\begin{array}{c}\mathbf{I}_{1}(0.6 \\
\text { IW/CPE) }\end{array}$ & $\begin{array}{c}\mathbf{I}_{\mathbf{2}}(\mathbf{0 . 8} \\
\text { IW/CPE) }\end{array}$ & $\begin{array}{c}\mathbf{I}_{3}(1.0 \\
\text { IW/CPE })\end{array}$ & & $\begin{array}{c}\text { I }_{1}(0.6 \\
\text { IW/CPE) }\end{array}$ & $\begin{array}{c}\mathbf{I}_{2}(0.8 \\
\text { IW/CPE) }\end{array}$ & $\begin{array}{c}\mathbf{I}_{3}(1.0 \\
\text { IW/CPE })\end{array}$ & \\
\hline Pg-Gypsum@500 kg ha ${ }^{-1}$ at flower initiation & 14.5 & 13.8 & 12.6 & 13.7 & 39.6 & 41.5 & 40.8 & 40.6 \\
\hline $\mathrm{Pg}_{2}$-PG @ $250 \mathrm{~kg} \mathrm{ha}^{-1}$ at flower initiation & 12.5 & 12.7 & 14.7 & 13.3 & 40.1 & 39.8 & 40.4 & 40.1 \\
\hline $\mathrm{Pg}_{3^{-}}$PG @ $250 \mathrm{~kg} \mathrm{ha}^{-1} 1 / 2$ as basal and $1 / 2$ at flower initiation & 12.2 & 13.0 & 13.7 & 13.0 & 37.7 & 39.7 & 40 & 39.1 \\
\hline $\mathrm{Pg}_{4^{-}}$PG @ $500 \mathrm{~kg} \mathrm{ha}^{-1 / 1 / 2}$ as basal and $1 / 2$ at flower initiation & 12.6 & 14.1 & 13.5 & 13.4 & 40.7 & 40.3 & 41.4 & 40.8 \\
\hline $\mathrm{Pg}_{5^{-}}$PG @ $500 \mathrm{~kg} \mathrm{ha}^{-1}$ at flower initiation & 13.3 & 14.7 & 15.8 & 14.6 & 41.2 & 41.1 & 41.7 & 41.3 \\
\hline MEAN & 13.0 & 13.7 & 14.1 & 13.6 & 39.8 & 40.5 & 40.8 & 40.4 \\
\hline \multicolumn{9}{|l|}{ Sub treatments at same level of main treatments } \\
\hline SEm \pm & & & & 0.3 & & & & 0.2 \\
\hline $\mathrm{CD}(\mathrm{P}=0.05)$ & & & & 0.8 & & & & 0.8 \\
\hline \multicolumn{9}{|l|}{ Main treatments at same or different level of sub treatments } \\
\hline SEm \pm & & & & 0.3 & & & & 0.3 \\
\hline $\mathrm{CD}(\mathrm{P}=0.05)$ & & & & 0.8 & & & & 0.8 \\
\hline
\end{tabular}


Table.2 Oil content and Oil yield of rabi groundnut as influenced by moisture regimes and phosphogypsum levels

\begin{tabular}{|c|c|c|}
\hline TREATMENTS & Oil content $(\%)$ & Oil Yield $\left(\mathrm{kg} \mathrm{ha}^{-1}\right)$ \\
\hline \multicolumn{3}{|l|}{ MOISTURE REGIMES (I) } \\
\hline $\mathrm{I}_{1}-0.6 \mathrm{IW} / \mathrm{CPE}$ & 39.8 & 1131 \\
\hline $\mathrm{I}_{2}=0.8 \mathrm{IW} / \mathrm{CPE}$ & 40.5 & 1417 \\
\hline $\mathrm{I}_{3}-1.0 \mathrm{IW} / \mathrm{CPE}$ & 40.9 & 1500 \\
\hline SEm \pm & 0.1 & 14.3 \\
\hline $\mathrm{CD}(\mathrm{P}=0.05)$ & 0.3 & 55.6 \\
\hline \multicolumn{3}{|l|}{ PHOSPHOGYPSUM LEVELS (Pg) } \\
\hline Pg1-Gypsum@ $500 \mathrm{~kg} \mathrm{ha}^{-1}$ at flower initiation & 40.6 & 1410 \\
\hline $\mathrm{Pg}_{2}-\mathrm{PG} @ 250 \mathrm{~kg} \mathrm{ha}^{-1}$ at flower initiation & 40.1 & 1315 \\
\hline $\mathrm{Pg}_{3^{3}}$ PG @ $250 \mathrm{~kg} \mathrm{ha}^{-1 / 1 / 2}$ as basal and $1 / 2$ at flower initiation & 39.1 & 1169 \\
\hline $\mathrm{Pg}_{4}$ - PG @ $500 \mathrm{~kg} \mathrm{ha}^{-11} 1 / 2$ as basal and $1 / 2$ at flower initiation & 40.8 & 1345 \\
\hline $\mathrm{Pg}_{5^{-}}$PG @ $500 \mathrm{~kg} \mathrm{ha}^{-1}$ at flower initiation & 41.3 & 1508 \\
\hline SEm \pm & 0.2 & 20.8 \\
\hline $\mathrm{CD}(\mathrm{P}=\mathbf{0 . 0 5})$ & 0.5 & 60.8 \\
\hline \multicolumn{3}{|l|}{ INTERACTION (I x Pg) } \\
\hline \multicolumn{3}{|l|}{ Sub treatment at same level of main treatment } \\
\hline SEm \pm & 0.2 & 31.9 \\
\hline $\mathrm{CD}(\mathrm{P}=0.05)$ & 0.8 & NS \\
\hline \multicolumn{3}{|l|}{ Main treatment at same or different level of sub treatment } \\
\hline SEm \pm & 0.3 & 35.3 \\
\hline $\mathrm{CD}(\mathrm{P}=0.05)$ & 0.8 & NS \\
\hline
\end{tabular}

Table.3 Effect of moisture regimes and phosphogypsum levels on economics of rabi groundnut

\begin{tabular}{|c|c|c|c|c|}
\hline TREATMENTS & $\begin{array}{c}\text { Cost of cultivation } \\
\qquad\left(\square \mathbf{h a}^{-1}\right)\end{array}$ & $\begin{array}{l}\text { Gross returns } \\
\quad\left(\square \mathbf{h a}^{-1}\right)\end{array}$ & $\begin{array}{l}\text { Net Returns } \\
\quad\left(\square \mathbf{h a}^{-1}\right)\end{array}$ & $\mathrm{B}:$ C ratio \\
\hline \multicolumn{5}{|l|}{ MOISTURE REGIMES (I) } \\
\hline$I_{1}-0.6 \mathrm{IW} / \mathrm{CPE}$ & 29462 & 59228 & 29767 & 2.0 \\
\hline $\mathrm{I}_{2}-0.8 \mathrm{IW} / \mathrm{CPE}$ & 29709 & 70751 & 41042 & 2.4 \\
\hline $\mathrm{I}_{3}-1.0 \mathrm{IW} / \mathrm{CPE}$ & 30080 & 72999 & 42909 & 2.4 \\
\hline SEm \pm & - & 625 & 625 & - \\
\hline $\mathrm{CD}(\mathrm{P}=0.05)$ & - & 2442 & 2442 & - \\
\hline \multicolumn{5}{|l|}{ PHOSPHOGYPSUM LEVELS (Pg) } \\
\hline $\begin{array}{l}\text { Pg }_{1} \text {-Gypsum@ } 500 \mathrm{~kg} \mathrm{ha}^{-1} \text { at flower } \\
\text { initiation }\end{array}$ & 300847 & 70022 & 39175 & 2.3 \\
\hline $\mathrm{Pg}_{2}$-PG @ $250 \mathrm{~kg} \mathrm{ha}^{-1}$ at flower initiation & 29104 & 66555 & 37451 & 2.3 \\
\hline $\begin{array}{l}\mathrm{Pg}_{3} \text { - PG @ } 250 \mathrm{~kg} \mathrm{ha}^{-1} 1 / 2 \text { as basal and } 1 / 2 \\
\text { at flower initiation }\end{array}$ & 29104 & 61036 & 31932 & 2.1 \\
\hline $\begin{array}{l}\mathrm{Pg}_{4} \text { - PG @ } 500 \mathrm{~kg} \mathrm{ha}^{-1} 1 / 2 \text { as basal and } 1 / 2 \\
\text { at flower initiation }\end{array}$ & 29848 & 68043 & 38195 & 2.3 \\
\hline $\mathrm{Pg}_{5^{-}}$PG @ $500 \mathrm{~kg} \mathrm{ha}^{-1}$ at flower initiation & 29848 & 72624 & 42776 & 2.4 \\
\hline SEm \pm & - & 690 & 690 & - \\
\hline $\mathrm{CD}(\mathrm{P}=0.05)$ & - & 2014 & 2014 & - \\
\hline \multicolumn{5}{|l|}{ INTERACTION (I x Pg) } \\
\hline \multicolumn{5}{|l|}{ Sub treatment at same level of main treatment } \\
\hline SEm \pm & - & 1398 & 1398 & - \\
\hline $\mathrm{CD}(\mathrm{P}=0.05)$ & - & 3788 & 3788 & - \\
\hline \multicolumn{5}{|c|}{ Main treatment at same or different level of sub treatment } \\
\hline SEm \pm & - & 1238 & 1238 & - \\
\hline $\mathrm{CD}(\mathrm{P}=0.05)$ & - & 3926 & 3926 & - \\
\hline
\end{tabular}

Cost of pods: Rs. $34 \mathrm{~kg}^{-1}$ 
Table.3a Gross returns and Net Returns of rabi groundnut as influenced by interaction between moisture regimes and Phosphogypsum levels

\begin{tabular}{|c|c|c|c|c|c|c|c|c|}
\hline \multirow{3}{*}{ PHOSPHOGYPSUM LEVELS } & \multicolumn{4}{|c|}{ Gross returns ( $\left.\square \mathbf{h a}^{-1}\right)$} & \multicolumn{4}{|c|}{ Net Returns ( $\square$ hä $^{-1}$ ) } \\
\hline & \multicolumn{3}{|c|}{ MOISTURE REGIMES } & \multirow[t]{2}{*}{ MEAN } & \multicolumn{3}{|c|}{ MOISTURE REGIMES } & \multirow[b]{2}{*}{ MEAN } \\
\hline & $\begin{array}{c}\mathrm{I}_{1}(0.6 \\
\text { IW/CPE })\end{array}$ & $\begin{array}{c}\mathrm{I}_{2}(0.8 \\
\mathrm{IW} / \mathrm{CPE})\end{array}$ & $\begin{array}{c}I_{3}(1.0 \\
\text { IW/CPE })\end{array}$ & & $\begin{array}{l}I_{1}(0.6 \\
\text { IW/CPE })\end{array}$ & $\begin{array}{c}\mathbf{I}_{2}(0.8 \\
\text { IW/CPE })\end{array}$ & $\begin{array}{c}\mathbf{I}_{3}(1.0 \\
\text { IW/CPE })\end{array}$ & \\
\hline $\begin{array}{l}\text { Pg }_{1}-\text { Gypsum @ } 500 \mathrm{~kg} \mathrm{ha}^{-1} \text { at flower } \\
\text { initiation }\end{array}$ & 61894 & 72546 & 75628 & 70022 & 31334 & 41740 & 44451 & 39175 \\
\hline $\begin{array}{l}\mathrm{Pg}_{2} \text {-PG @ } 250 \mathrm{~kg} \mathrm{ha}^{-1} \text { at flower } \\
\text { initiation }\end{array}$ & 55548 & 70187 & 73932 & 66555 & 26732 & 41124 & 44498 & 37451 \\
\hline $\begin{array}{l}\mathrm{Pg}_{3} \text { PG @ } 250 \mathrm{~kg} \mathrm{ha}^{-1} 1 / 2 \text { as basal and } 1 / 2 \\
\text { at flower initiation }\end{array}$ & 53034 & 62819 & 67255 & 61036 & 24218 & 33756 & 37821 & 31932 \\
\hline $\begin{array}{l}\mathrm{Pg}_{4^{-}} \mathrm{PG} @ 500 \mathrm{~kg} \mathrm{ha}^{-1} 1 / 2 \text { as basal and } 1 / 2 \\
\text { at flower initiation }\end{array}$ & 60807 & 73496 & 69826 & 68043 & 31247 & 43689 & 39650 & 38195 \\
\hline $\begin{array}{l}\text { Pg }_{5^{-}} \text {PG @ } 500 \mathrm{~kg} \mathrm{ha}^{-1} \text { at flower } \\
\text { initiation }\end{array}$ & 64863 & 74707 & 78302 & 72624 & 35303 & 44900 & 48124 & 42776 \\
\hline MEAN & 59228 & 70751 & 72999 & 67656 & 29767 & 41041 & 42909 & 37906 \\
\hline \multicolumn{9}{|c|}{ Sub treatment at same level of main treatment } \\
\hline SEm \pm & & & & 1398 & & & & 1398 \\
\hline $\mathrm{CD}(\mathrm{P}=\mathbf{0 . 0 5})$ & & & & 3788 & & & & 3788 \\
\hline \multicolumn{9}{|c|}{ Main treatment at same or different level of sub treatment } \\
\hline SEm \pm & & & & 1238 & & & & 1238 \\
\hline $\mathrm{CD}(\mathrm{P}=\mathbf{0 . 0 5})$ & & & & 3926 & & & & 3926 \\
\hline
\end{tabular}


Fig.1 Effect of moisture regimes on pod yield and haulm yield $\left(\mathrm{kg} \mathrm{ha}^{-1}\right)$ of rabi groundnut

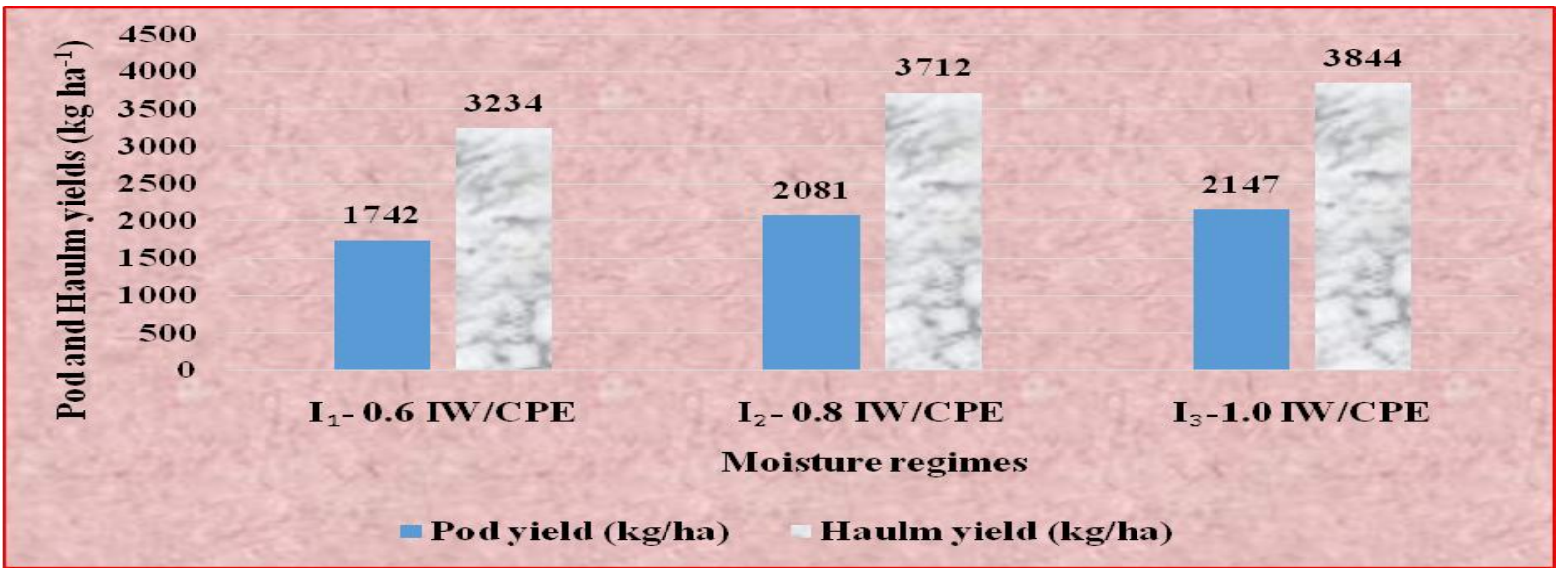

Fig.2 Effect of phosphogypsum levels on pod yield and haulm yield $\left(\mathrm{kg} \mathrm{ha}^{-1}\right)$ of $r a b i$ groundnut

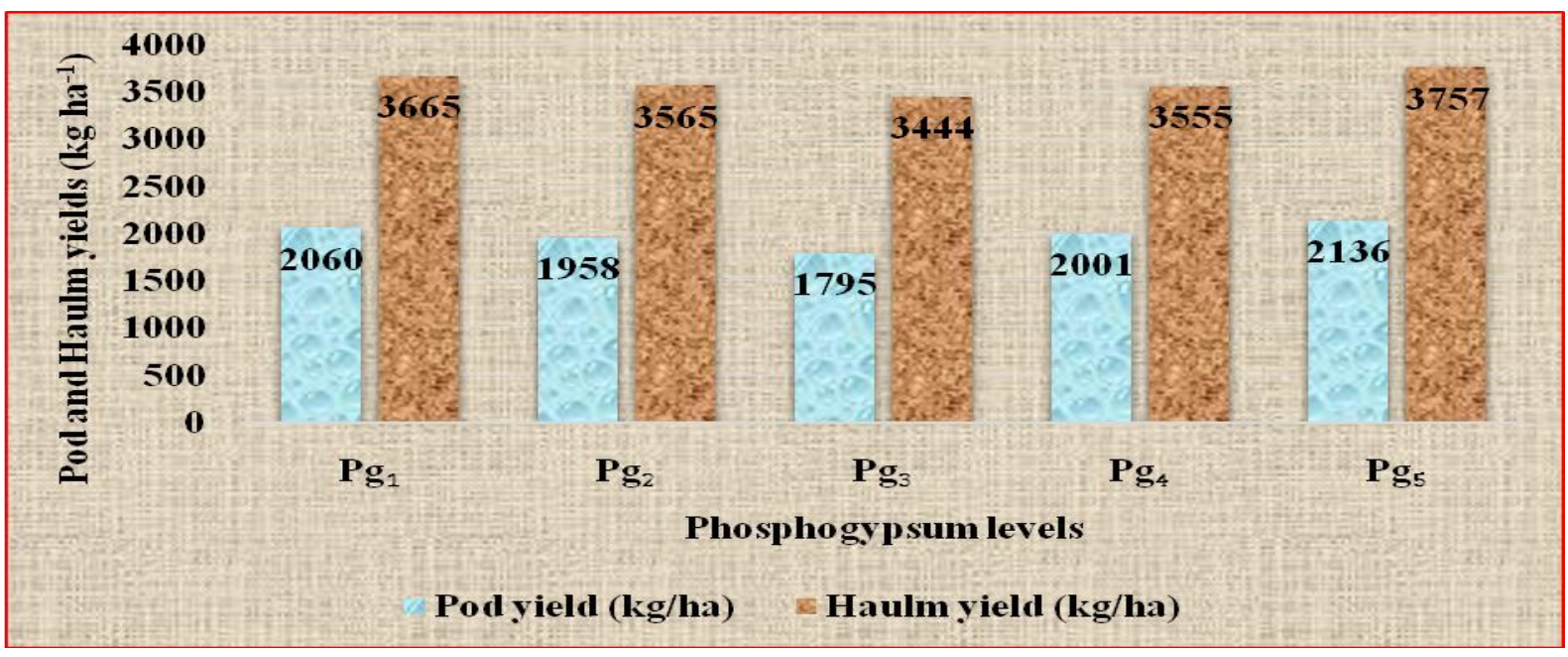

Fig.3 Interaction effect of moisture regimes and phosphogypsum levels on pod yield $\left(\mathrm{kg} \mathrm{ha}^{-1}\right)$

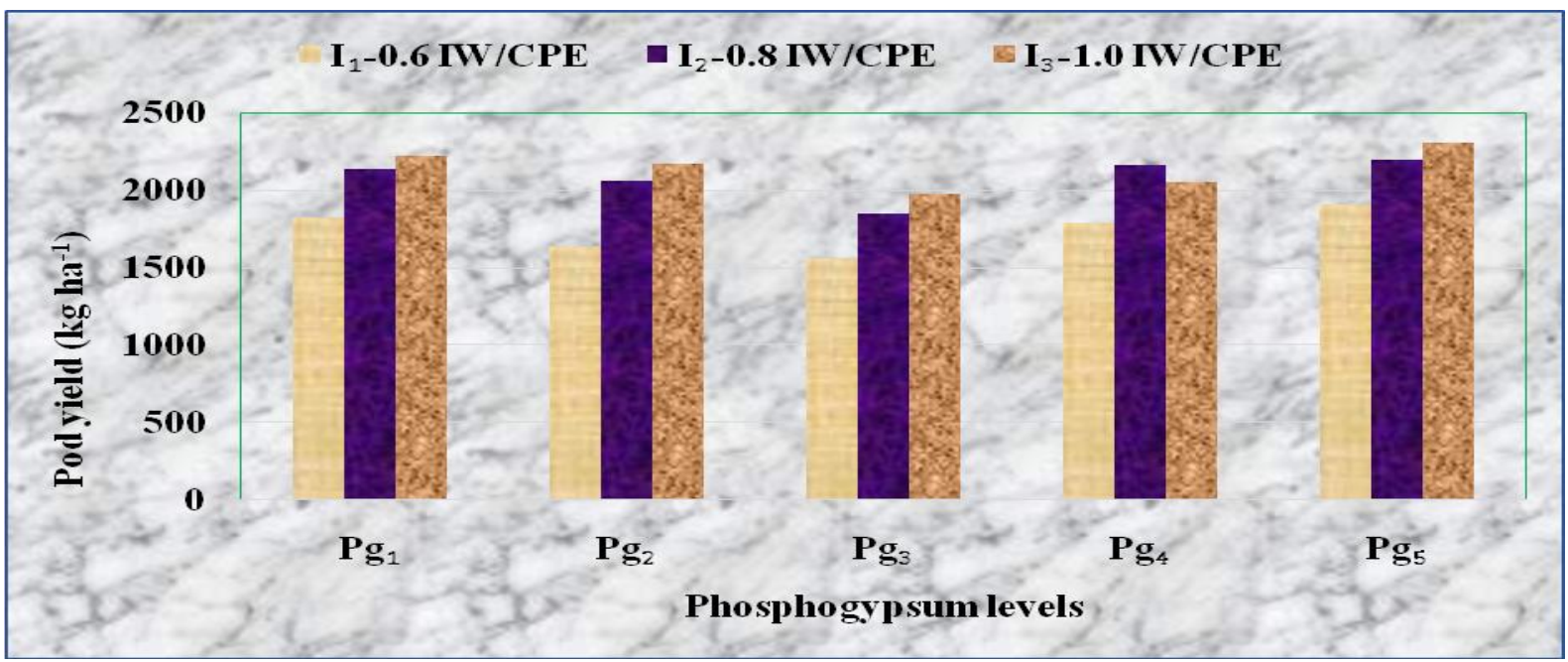


The interaction effect between moisture regimes and phosphogypsum levels was significant on both the parameters and recorded significantly highest pod yield $\left(23.03 \mathrm{q} \mathrm{ha}^{-1}\right)$ at interaction level $\mathrm{I}_{3} \mathrm{Pg}_{5}(1.0$ IW/CPE and phosphogypsum @ $500 \mathrm{~kg} \mathrm{ha}^{-1}$ at flower initiation), which was on par with $\mathrm{I}_{3} \mathrm{Pg}_{1}, \mathrm{I}_{2} \mathrm{Pg}_{5}, \mathrm{I}_{3} \mathrm{Pg}_{2}, \mathrm{I}_{2} \mathrm{Pg}_{4}, \mathrm{I}_{2} \mathrm{Pg}_{1}, \mathrm{I}_{3} \mathrm{Pg}_{4}$ and $\mathrm{I}_{2} \mathrm{Pg}_{2}$. Lowest pod yield (15.6 q ha $\left.{ }^{-1}\right)$ obtained at interaction level $\mathrm{I}_{1} \mathrm{Pg}_{3}(0.6 \mathrm{IW} / \mathrm{CPE}$ and phosphogypsum @ $250 \mathrm{~kg} \mathrm{ha}^{-1} 1 / 2$ as basal and $1 / 2$ at flower initiation). Similarly highest haulm yield $\left(39.85 \mathrm{q} \mathrm{ha}^{-1}\right)$ obtained at interaction $\mathrm{I}_{2} \mathrm{Pg}_{5}$ followed by $\mathrm{I}_{3} \mathrm{Pg}_{2}, \mathrm{I}_{2} \mathrm{Pg}_{1}$ and $\mathrm{I}_{3} \mathrm{Pg}_{1}$ interactions respectively. Lowest haulm yield $\left(30.46 \quad \mathrm{q} \mathrm{ha}^{-1}\right)$ observed at $\mathrm{I}_{1} \mathrm{Pg}_{2}$ interaction.

The data pertaining to groundnut oil content and oil yield were presented in Table 2 . Significantly the highest oil content and oil yield $\left(40.9 \%\right.$ and $\left.1500 \mathrm{~kg} \mathrm{ha}^{-1}\right)$ were obtained with moisture regimes at $\mathrm{I}_{3}(1.0 \mathrm{IW} / \mathrm{CPE})$ over other treatments, while lowest values $(39.8 \%$ and $\left.1131 \mathrm{~kg} \mathrm{ha}^{-1}\right)$ were recorded in $\mathrm{I}_{1}(0.6$ IW/CPE) treatment. These results were in close confirmity with the findings of Somnath Chattopaddhyay and Goutam Kumar Ghosh (2012), Patel et al., (2009).

Among phosphogypsum levels, application of phosphogypsum @ $500 \mathrm{~kg} \mathrm{ha}^{-1}$ at flower initiation was recorded highest oil content and oil yield $\left(41.3 \%\right.$ and $\left.1508 \mathrm{~kg} \mathrm{ha}^{-1}\right)$ of groundnut, while oil content was on par with the gypsum application @ $500 \mathrm{~kg} \mathrm{ha}^{-1}$ at flower initiation $\left(\mathrm{Pg}_{1}\right)$ but significantly superior than other treatments. The lowest oil content and oil yield (39.1\% and $\left.1169 \mathrm{~kg} \mathrm{ha}^{-1}\right)$ were obtained under $\mathrm{Pg}_{3}$ (phosphogypsum $250 \mathrm{~kg} \mathrm{ha}^{-1} 1 / 2$ as basal and $1 / 2$ at flower initiation) treatment. The interaction effect between moisture regimes and phosphogypsum levels was significant on oil content. Significantly highest oil content recorded with interaction level of phosphogypsum @ $500 \mathrm{~kg} \mathrm{ha}^{-1}$ at flower initiation $\left(\mathrm{Pg}_{5}\right)$ irrespective levels of moisture regimes. Lowest oil content observed at interaction level $\mathrm{I}_{1} \mathrm{Pg}_{3}$ (Table 1.b). The increased oil content with phosphogypsum in groundnut might be associated with sulphur and calcium nutrition to groundnut. Sulphur is a constituent of protein and plays a pivotal role in oil synthesis. These results were in close confirmity with the findings of Patel et al., (2009) and Patil et al., (2003).

The data pertaining to groundnut economics was presented in Table 3. The economics of groundnut as influenced by moisture regimes and phosphogypsum levels revealed that highest gross returns $\left(72999 \square \mathrm{ha}^{-1}\right)$, net returns $\left(42909 \square \mathrm{ha}^{-1}\right)$ were recorded at $\mathrm{I}_{3}$ (IW/CPE 1.0) irrigation level with five irrigations over other treatments. Whereas the highest B: C ratio (2.4) was recorded at irrigation level IW/CPE 1.0 and 0.8 (five and four irrigations). The minimum gross returns $\left(59228 \square \mathrm{ha}^{-1}\right)$, net returns $\left(29767 \square \mathrm{ha}^{-1}\right)$ and B: C ratio (2.0) was obtained under IW/CPE 0.6 moisture regime with three irrigations. This finding was in confirmity with Rathod and Trivedi (2011), Patel et al., (2009), Raskar and Bhoi (2003).

Regarding the phosphogypsum levels, the maximum gross return $\left(72624 \square \mathrm{ha}^{-1}\right)$, net return $\left(42776 \square \mathrm{ha}^{-1}\right.$ ) and B: C ratio (2.4) was recorded at phosphogypsum application @ $500 \mathrm{~kg} \mathrm{ha}^{-1}$ at flower initiation $\left(\mathrm{Pg}_{5}\right)$ followed by $\mathrm{Pg}_{1}, \mathrm{Pg}_{2}$ and $\mathrm{Pg}_{4}$ treatments. This finding was in confirmity with Dash et al., (2013), Patel et al., (2009).

Interaction effect between moisture regimes and phosphogypsum on gross returns and net returns data (Table.3.a) indicated that the highest gross returns and net returns $\left(\square \mathrm{ha}^{-1}\right.$ 78302 and 48124) recorded with interaction level $\mathrm{I}_{3} \mathrm{Pg}_{5} \quad(1.0 \quad \mathrm{IW} / \mathrm{CPE}$ ratio and phosphogypsum@500 $\mathrm{kg} \mathrm{ha}^{-1}$ at flower 
initiation), which was on par with $\mathrm{I}_{3} \mathrm{Pg}_{1}, \mathrm{I}_{2} \mathrm{Pg}_{5}$ (gross returns) and $\mathrm{I}_{2} \mathrm{Pg}_{5}, \mathrm{I}_{3} \mathrm{Pg}_{2}, \mathrm{I}_{3} \mathrm{Pg}_{1}$ (net returns) respectively. Lowest gross returns and net returns recorded at interaction of $\mathrm{I}_{1} \mathrm{Pg}_{3}$ (0.6 IW/CPE and phosphogypsum @ $250 \mathrm{~kg}$ $\mathrm{ha}^{-1} 1 / 2$ as basal and $1 / 2$ at flower initiation).

It can be concluded from this study that for maximizing the production of groundnut, moisture regime at $\mathrm{I}_{2}(0.8 \mathrm{IW} / \mathrm{CPE})$ and phosphogypsum at $500 \mathrm{~kg} \mathrm{ha}^{-1}$ at flower initiation can be recommended in semiarid climate of Andhra Pradesh.

Scheduling irrigation at $\mathrm{I}_{3}(1.0 \mathrm{IW} / \mathrm{CPE})$ and application of phosphogypsum @ $500 \mathrm{~kg} \mathrm{ha}^{-1}$ at flower initiation recorded higher pod as well as haulm yield.

It is concluded from this study that irrigation with $\mathrm{I}_{2} \quad(0.8 \mathrm{IW} / \mathrm{CPE})$ and application of phosphogypsum @ $000 \mathrm{~kg} \mathrm{ha}^{-1}$ applied at flower initiation resulted in higher yield, quality and economic returns (B: C ratio 2.4) of rabi groundnut under semi- arid climate of Hyderabad.

The results of the experiment clearly suggest that phosphogypsum is also efficient in increasing the pod and haulm yield of groundnut similar to that of gypsum.

\section{References}

Bibhu Santosh behera, Mohit Das, Anama Charan Behera and Rudra Ashish behera 2015. Weather based irrigation scheduling in summer groundnut in Odisha condition. International journal of Agricultural Science and Research. 5(5): 247-260.

Biswas, P. P. and Sharma, P. D. 2008. Phosphogypsum a potential source of sulphur and calcium to crops. Indian Journal of Fertilizers. 4(8): 49-52.

Dash, A. K., Nayak, B. R., Panigrahy, N.,
Mohapatra, S. and Samant, P. K. 2013. Performance of groundnut (Arachis hypogaea L.) under different levels of sulphur and irrigation. Indian Journal of Agronomy. 58(4): 578-582.

Dey, J., Basu, T. K. and Bandyopadhyay, S. R. 2007. Effect of different levels of irrigation and sources of sulphur on nodulation and yield of groundnut. Journal of Mycopathology Research. 45(1): 73-75.

Giri, U., Paul, N., Giri, S., Bandyopadhyay, P. and Nanda M.K. 2017. Effect of sulphur and different irrigation regimes on PAR distribution, canopy temperature, yield and water use efficiency of Groundnut (Arachis hypogaea L.). International journal of Agriculture, Environment and Biotechnology. 10(2): 177-187.

Gosh, P. K., Hati, K. M., Mandal, K. G., Misra, A. K., Chaudhary, A. S. and Bandyopadhyay, K. K. 2000. Sulphur nutrition in oilseeds and oilseed-based cropping systems. Fertiliser News. 45(8): 27-40.

Panse, V. G. and Sukhatme, P. V. 1985. Statistical methods for agricultural workers. ICAR publication.

Patel, G. N., Patel, P. T., Patel, P. H., Patel, D. M., Patil, D. K. and Patil, R. M. 2009. Yield attributes, yield, quality and uptake of nutrients by summer groundnut, Arachis hypogaea $L$. as influenced by sources and levels of sulphur under varying irrigation schedules. Journal of Oilseeds Research. 26(2): 119-122.

Patil, S. S., Raut, P. D., Ingle, G. L. and Dongare, S. T. 2003. Effect of sulphur and magnesium on yield, nutrient uptake and quality of groundnut. New Agriculturist. 14 (1/2): 49-52.

Raskar, B. S. and Bhoi, P. G. 2003. Response of summer groundnut to irrigation regimes and mulching. Indian Journal of Agronomy. 48 (3): 210-213. 
Rathod, A. B. and Trivedi, S. A. 2011. Summer groundnut crop performance and economics under drip irrigation at various water application levels. National Conference on Recent Trends in Engineering \& Technology.

Rout, K. K. and Jena, D. 2009. Effect of phosphogypsum and its combined use with lime in acid soils of orissa on crop productivity, crop and soil quality. Indian Journal of Fertilizers. 5(5): 4454.

Shaikh, A. A., Nimbalkar, C. A. and Jawale, S. M. 2004. Effect of irrigation scheduling and mulches on yield and yield contributing characters of summer groundnut.

Journal of Maharashtra Agricultural $U$ niversity. 29(2): 163-166.

Somnath Chattopaddhyay and Goutam kumar
Ghosh. 2012. Response of rapeseed to various sources and levels of sulphur in red and lateritic soils of west Bengal, India. International Journal of plant, Animal and Environmental sciences. 2(4): 50-59.

Surendra singh and Singh, S.K. 2016. Use of indigenous sources of sulphur in soils of eastern India for higher crops yield and quality: A review. Agricultural Reviews. 37(2): 117-124.

Suresh, K., Balaguravaiah, D., Ramulu, V. and Sujani rao, C. H. 2013. Comparative efficiency of sprinkler irrigation over check basin irrigation in groundnut at different irrigation schedules. International journal of plant, animal and environmental sciences. 3(2): 9-13.

\section{How to cite this article:}

Naresha, R., P. Laxminarayana, K.B. Suneetha Devi and Sailaja, V. 2018. Quality, Yield and Economics of Rabi Groundnut as Influenced by Irrigation Scheduling and Phosphogypsum Levels. Int.J.Curr.Microbiol.App.Sci. 7(03): 3292-3303. doi: https://doi.org/10.20546/ijcmas.2018.703.379 